\title{
Passive Series-Parallel Compensator for Non-sinusoidal Power System
}

\author{
Seong-Jeub Jeon \\ Department of Electronic Engineering \\ Pukyong National University \\ 45 Yongsoro, Nam-Gu, 48513 Busan KOREA \\ Phone/Fax number:+82 51629 6216/6210, e-mail: jeub@pknu.ac.kr
}

\begin{abstract}
In this paper, a simple but clear method finding an L-C network satisfying reactances or susceptances at given frequencies is proposed. Applying this method to synthesizing series-parallel compensator, unity power factor can be obtained when frequency-dependent impedances of nonlinear load are given. A series-parallel compensator can also be used to isolate a load from a source with respect to harmonics.
\end{abstract}

\section{Key words}

L-C network, Series-parallel Compensation, Reactive Power, Transmission Loss, Non-sinusoidal.

\section{Introduction}

Since Lyon reported that the current in an ac system cannot be optimum [1], reactive power has been blamed for increased transmission losses, and reactive power compensation at the load side has been considered very important because it minimizes transmission loss [2-10]. In transmision systems, a series compensation is effective and being used [2]. In a load side, parallel compensation is being used widely. The advent of power electronics made harmonics proliferous in power systems. More complicated and sophistigated compensator is needed [310]. In this paper, a series-parallel compensation which is very effective in compensating a nonlinear load whose impedances are given and in isolating a power source from a harmonic-polluted load, is studied. A seriesparallel compensation has been studied by Prof. Czarnecki for a load side compensation [9], [10]. However, the explanation about the principle and the process of seriesparallel compensation is not satisfactory. In this paper, two kinds of L-C circuits are studied as building blocks for series-parallel compensator: series L-C circuits in parallel and parallel L-C circuits in series. A simple and systematic design method for an L-C network (a building block) satisfying reactances or susceptances at given frequencies is given, which is a basis for a design of the series-parallel compensator.

\section{Power analysis of harmonic polluted system}

A simple frequency-domain interpretation of reactive power in Fig 1 is possible using Fourier analysis. An orthogonal decomposition of the voltages and the currents, assuming periodicity, is given as follows [11], [12]:

$$
\begin{aligned}
& v_{s}=\sum_{k=1}^{\infty}\left(v_{2 k-1}+v_{2 k}\right), \\
& i_{s}=\sum_{k=1}^{\infty}\left(i_{2 k-1}+i_{2 k}\right) \quad \text { for } l=1,2, \ldots, m,
\end{aligned}
$$

where

$$
\begin{aligned}
& v_{2 k-1}=\sqrt{2} V_{2 k-1} \sin k \omega t, v_{2 k}=\sqrt{2} V_{2 k} \cos k \omega t \\
& i_{2 k-1}=\sqrt{2} I_{2 k-1} \sin k \omega t, i_{2 k}=\sqrt{2} I_{2 k} \cos k \omega t .
\end{aligned}
$$

$V_{j}$ and $I_{j}$ are signed rms values of $v_{j}$ and $i_{j}$ respectively. Their absolute values are rms values and their signs are dependent on the directions of voltage and current references shown in Fig. 1. Two components, $\left\{v_{i}, i_{i}\right\}$ and $\left\{v_{j}, i_{j}\right\}$, are considered orthogonal to each other because the products, $v_{i} i_{j}$ and $v_{j} i_{i}$, do not produce average power over a period. The average power over a period is given by

$$
P=\sum_{j=1}^{\infty} V_{j} I_{j}
$$

The effective current and the effective voltage are defined by

$$
I_{\mathrm{e}} \equiv \sqrt{\sum_{j=1}^{\infty} I_{j}^{2}}, \quad V_{\mathrm{e}} \equiv \sqrt{\sum_{j=1}^{\infty} V_{j}^{2}} .
$$

The transmission loss is proportional to the square of the effective current $I_{\mathrm{e}}$. The apparent power and the reactive power is defined by

$$
S \equiv V_{\mathrm{e}} I_{\mathrm{e}}, Q \equiv \sqrt{S^{2}-P^{2}}
$$




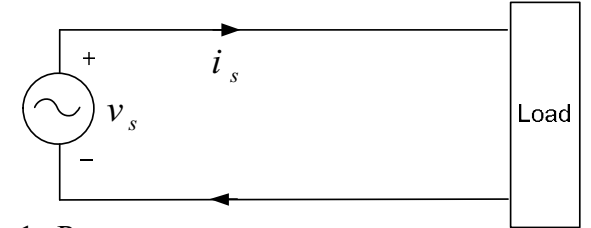

Fig. 1. Power system.

The condition for achieving unity power factor is that the currents must have the same spectral distribution as the voltages in order to achieve unity power factor. It is known that currents yielding average power $P$ with zero reactive power is given by

$$
I_{i}=\frac{P}{V_{\mathrm{e}}^{2}} V_{i} \text { for all } i \text {. }
$$

Multiplying $V_{i}$ to both sides,

$$
P_{i}=\frac{P}{V_{\mathrm{e}}^{2}} V_{i}^{2} \text { for all } i
$$

Combining $2 k$ th and $(2 k+1)$ th components, harmonic power for $\omega_{k}$ can be obtained:

$$
P^{\omega_{k}}=P_{2 k}+P_{2 k+1}=\frac{P}{V_{e}^{2}}\left(V_{2 k}^{2}+V_{2 k+1}^{2}\right)=\frac{P}{V_{e}^{2}}\left(V^{\omega_{k}}\right)^{2} .
$$

The superscripts denote the angular frequency of the harmonic.

\section{Compensation of power system}

The series-parallel compensator can be used in a power system to enhance power factors by compensating circuits. The series-parallel compensator can be applied to three specific cases: 1) when impedance of frequencydependent load is given, 2) when harmonic powers of load are given and 3) when harmonic isolation between a power source and a load is needed.

3.1 Compensation when load impedance and total power are given

Some nonlinear load such as discharge lamp can be modelled as a frequency-dependent impedance. Power system with this kind of load can have unity power factor when compensated with series-parallel compensator as shown in Fig. 2. The series-parallel compensator can distribute the harmonic power to satisfy unity power factor condition. To be unity power factor at source side, the power should be distributed as follows:

$$
P_{\mathrm{s}}^{\omega_{i}}=\frac{P}{V_{\mathrm{se}}^{2}}\left|\mathbf{V}_{\mathrm{s}}^{\omega_{i}}\right|^{2} \text {. }
$$

The superscripts of current and voltage phasors denote the angular frequency of the phasor. Each harmonic current should be as follows:

$$
\mathbf{I}_{\mathrm{s}}^{\omega_{i}}=\frac{P}{V_{\mathrm{se}}^{2}} \mathbf{V}_{\mathrm{s}}^{\omega_{i}}
$$

The compensator, placed between the source and the load, is a linear reactive circuit where power is neither generated nor dissipated for each harmonic. Accordingly, the harmonic power at load side is equal to that at source side:

$$
P_{\mathrm{L}}^{\omega_{i}}=P_{\mathrm{s}}^{\omega_{i}}=\frac{P}{V_{\mathrm{se}}^{2}}\left|\mathbf{V}_{\mathrm{s}}^{\omega_{i}}\right|^{2} .
$$

Being the load impedance given, the magnitude of load voltage for each harmonic is given by

$$
\left|\mathbf{V}_{\mathrm{L}}^{\omega_{i}}\right|^{2}=\frac{P_{\mathrm{L}}^{\omega_{i}}}{G_{\mathrm{L}}\left(\omega_{i}\right)} .
$$

From the phasor diagram in Fig. 2(b), the reactance of the series element is given by

$$
X_{\mathrm{S}}^{\omega_{i}}=\frac{V_{\mathrm{se}}^{2}}{P} \sqrt{\left|\frac{\mathbf{V}_{\mathrm{L}}^{\omega_{i}}}{\mathbf{V}_{\mathrm{s}}^{\omega_{i}}}\right|^{2}-1} .
$$

And the phasor voltage across the load is given by

$$
\mathbf{V}_{\mathrm{L}}^{\omega_{i}}=\mathbf{V}_{\mathrm{s}}^{\omega_{i}}-j X_{\mathrm{S}} \mathbf{I}_{\mathrm{s}}^{\omega_{i}} .
$$

$\mathbf{V}_{\mathrm{L}}^{\omega_{i}}$ can be obtained by substituting (13) into (14). The load current is given by

$$
\mathbf{I}_{\mathrm{L}}^{\omega_{i}}=\frac{\mathbf{V}_{\mathrm{L}}^{\omega_{i}}}{\mathbf{Z}_{\mathrm{L}}\left(\omega_{i}\right)}
$$

The compensation current is given by

$$
\mathbf{I}_{\mathrm{C}}^{\omega_{i}}=\mathbf{I}_{\mathrm{L}}^{\omega_{i}}-\mathbf{I}_{\mathrm{s}}^{\omega_{i}} \text {. }
$$

The susceptance of the parallel element is given by

$$
B_{\mathrm{P}}^{\omega_{i}}=\frac{j \mathbf{I}_{\mathrm{C}}^{\omega_{i}}}{\mathbf{V}_{\mathrm{L}}^{\omega_{i}}}
$$

Thus, series-parallel compensator is synthesized.

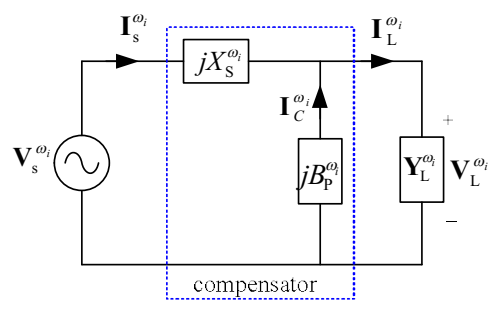

(a)

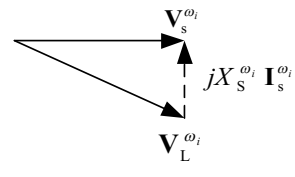

(b)
Fig. 2. Series-parallel compensator: (a) circuit diagram, (b) its phasor diagram

3.2 Compensation when harmonic powers and their voltages are given

Harmonic powers as in a thyristor rectifier are thought given. There is no power redistribution among frequencies when an L-C network is used. Therefore, the seriesparallel compensator could not redistribute harmonic powers, Each harmonic power at the load side is conserved at the source side, i.e.

$$
P_{\mathrm{s}}^{\omega_{i}}=P_{\mathrm{L}}^{\omega_{i}}
$$

Each harmonic current should be as follows: 


$$
\mathbf{I}_{\mathrm{s}}^{\omega_{i}}=\frac{P_{\mathrm{s}}^{\omega_{i}}}{\left|\mathbf{V}_{\mathrm{s}}^{\omega_{i}}\right|^{2}} \mathbf{V}_{\mathrm{s}}^{\omega_{i}} \text { for all } i
$$

Considering Fig. 2, the series reactive component is given by

$$
X_{\mathrm{S}}^{\omega_{i}}=\frac{1}{\left|\mathbf{I}_{\mathrm{s}}^{\omega_{i}}\right|} \sqrt{\left|\mathbf{V}_{\mathrm{L}}^{\omega_{i}}\right|^{2}-\left|\mathbf{V}_{\mathrm{s}}^{\omega_{i}}\right|^{2}}
$$

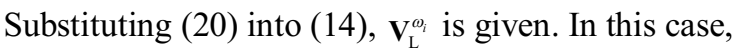
power is given, so $\mathbf{I}_{\mathrm{L}}^{\Phi_{\mathrm{i}}}$ is given by

$$
\mathbf{I}_{\mathrm{L}}^{\omega_{i}}=\left(\frac{P_{\mathrm{L}}^{\omega_{i}}+j Q_{\mathrm{L}}^{\omega_{i}}}{\mathbf{V}_{\mathrm{L}}^{\omega_{i}}}\right)^{*} .
$$

Equations (16) and (17) is still valid in this case.

\subsection{Compensation Circuit Synthesis}

In a case with two frequencies, the circuits in Fig. 3 can be considered for a single element in Fig. 2. The susceptance variations are shown in Fig. 3. When the circuit in Fig. 3(a) is used, the susceptances $B\left(\omega_{1}\right)$ and $B\left(\omega_{2}\right)$ at the two frequencies can be satisfied with the following circuit parameters:

$$
\begin{gathered}
C=\frac{B\left(\omega_{1}\right) B\left(\omega_{2}\right)\left(\omega_{2}^{2}-\omega_{1}^{2}\right)}{\omega_{1} \omega_{2}\left(\omega_{2} B\left(\omega_{2}\right)-\omega_{1} B\left(\omega_{1}\right)\right)} \\
L=\frac{\omega_{1} B\left(\omega_{2}\right)-\omega_{2} B\left(\omega_{1}\right)}{B\left(\omega_{1}\right) B\left(\omega_{2}\right)\left(\omega_{2}^{2}-\omega_{1}^{2}\right)} .
\end{gathered}
$$

where $\omega_{2}>\omega_{1}$ is assumed. For the circuit parameters to be positive the following conditions should be satisfied:

$$
\begin{aligned}
& \frac{B\left(\omega_{2}\right)}{B\left(\omega_{1}\right)}>\frac{\omega_{2}}{\omega_{1}} \text { for positive susceptances } \\
& \frac{B\left(\omega_{2}\right)}{B\left(\omega_{1}\right)}<\frac{\omega_{1}}{\omega_{2}} \text { for negative susceptances. }
\end{aligned}
$$

If $B\left(\omega_{1}\right)$ is negative and $B\left(\omega_{2}\right)$ is positive, this circuit cannot be used. If $B\left(\omega_{1}\right)$ is positive and $B\left(\omega_{2}\right)$ is negative, there is no restrictive condition, i.e. any susceptances can be satisfied.

When the circuit in Fig. 3(b) is used, the susceptances $B\left(\omega_{1}\right)$ and $B\left(\omega_{2}\right)$ at the two frequencies can be satisfied with the following circuit parameters:

$$
\begin{aligned}
& C=\frac{\omega_{2} B\left(\omega_{2}\right)-\omega_{1} B\left(\omega_{1}\right)}{\omega_{2}^{2}-\omega_{1}^{2}} \\
& L=\frac{\omega_{2}^{2}-\omega_{1}^{2}}{\omega_{1} \omega_{2}\left(\omega_{1} B\left(\omega_{2}\right)-\omega_{2} B\left(\omega_{1}\right)\right)} .
\end{aligned}
$$

For the circuit parameters to be positive, the conditions (24) and (25) should be satisfied.

If $B\left(\omega_{1}\right)$ is positive and $B\left(\omega_{2}\right)$ is negative, this circuit cannot be used. If $B\left(\omega_{1}\right)$ is negative and $B\left(\omega_{2}\right)$ is positive, there is no restrictive condition.

For two or more frequencies, multiple series-connected circuits in Fig. 3(a) can be parallel connected as shown in Fig. 5(a). Multiple parallel-connected circuits in Fig. 3(b) can be series connected for the same purpose. The circuit in Fig. 5 was intensively studied by Czarnecki. However, an easier and more systematic design method is given here. The susceptances can be satisfied by placing resonant frequencies near the frequencies at which the susceptances should be satisfied. If the susceptance is positive, the resonance frequency will be slightly higher, and if the susceptance is negative, the resonance frequency is slightly lower than the frequency. Total $n$ L-C series circuits can satisfy the required susceptances at $n$ frequencies. Under certain conditions, some of them can be omitted. However, $n$ circuits are used for $n$ frequencies in this paper. The total susceptance is the sum of the susceptances of the individual L-C circuits.

$$
B(\omega)=\sum_{k} \frac{\omega C_{k}}{1-\omega^{2} L_{k} C_{k}}=\sum_{k} \frac{\omega C_{k}}{1-\left(\omega / \omega_{r k}\right)^{2}} .
$$

where

$$
\omega_{r k}^{2}=\frac{1}{L_{k} C_{k}} .
$$

Let us make a susceptance vector, $\mathbf{B}$, gathering susceptances to be satisfied; and a capacitance vector, $\mathbf{C}$, gathering capacitances comprising L-C circuits.

$$
\begin{aligned}
& \mathbf{B}=\left[B\left(\omega_{1}\right) B\left(\omega_{2}\right) \cdots B\left(\omega_{k}\right) \cdots B\left(\omega_{n}\right)\right]^{T} \\
& \mathbf{C}=\left[C_{1} C_{2} \cdots C_{k} \cdots C_{n}\right]^{T} .
\end{aligned}
$$

Then, (28) is expressed as follows:

$$
B=A C
$$

where

$$
\begin{aligned}
\mathbf{A} & =\left[a_{i j}\right]_{n \times n} \\
a_{i j} & =\frac{\omega_{i}}{1-\left(\omega_{i} / \omega_{r j}\right)^{2}} .
\end{aligned}
$$

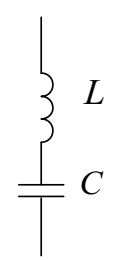

(a)

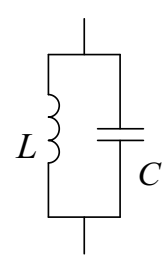

(b)
Fig. 3. Circuits adoptable in case of two frequencies (a) series L-C circuit, (b) parallel L-C circuit

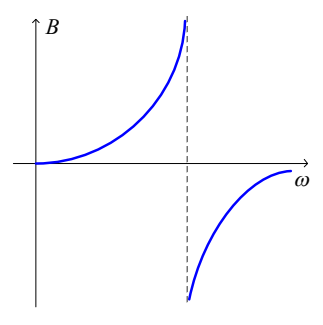

(a)

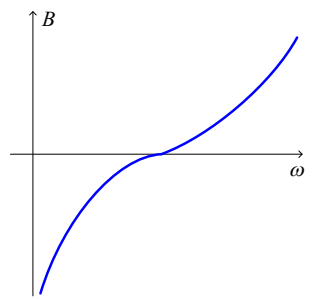

(b)
Fig. 4. Variations of susceptances of the circuits in Fig. 3

(a) susceptance of Fig. 3(a), (b) susceptance of Fig. 3(b) 
The capacitance vector is determined by

$$
\mathbf{C}=\mathbf{A}^{-1} \mathbf{B} \text {. }
$$

The inductances can be determined by applying capacitances obtained by (35) into (29). Thus, the L-C network satisfying susceptances at given frequencies is synthesized.

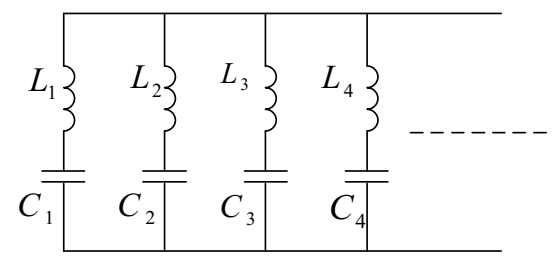

(a)

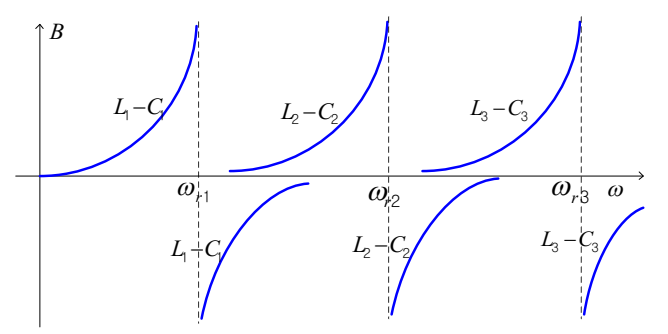

(b)

Fig. 5. Parallel connection of L-C series circuits

(a) circuit diagram, (b) its susceptance variation

The circuit in Fig. 6(a) can be adopted for the same purpose. For this circuit, it is convenient to consider reactances. The reactances can be satisfied by placing resonant frequencies near the frequencies at which the reactances should be satisfied. The resonance frequency will be slightly higher if the reactance is positive, and the resonance frequency is slightly lower than the frequency if the reactance is negative. Total $n \mathrm{~L}-\mathrm{C}$ parallel circuits can satisfy the required reactances at $n$ frequencies. Under certain conditions, some of them can be omitted. However, $n$ circuits are used for $n$ frequencies in this paper. The total reactance is the sum of the reactances of the individual L-C circuits:

$$
X(\omega)=\sum_{k} \frac{\omega L_{k}}{1-\omega^{2} L_{k} C_{k}}=\sum_{k} \frac{\omega L_{k}}{1-\left(\omega / \omega_{r k}\right)^{2}}
$$

where

$$
\omega_{r k}^{2}=\frac{1}{L_{k} C_{k}} .
$$

Let us make a reactance vector $\mathbf{X}$, gathering reactances to be satisfied; and an inductance vector $\mathbf{L}$, gathering inductances comprising L-C circuits.

$$
\begin{aligned}
& \mathbf{X}=\left[X\left(\omega_{1}\right) X\left(\omega_{2}\right) \cdots X\left(\omega_{k}\right) \cdots X\left(\omega_{n}\right)\right]^{T} \\
& \mathbf{L}=\left[L_{1} L_{2} \cdots L_{k} \cdots L_{n}\right]^{T} .
\end{aligned}
$$

Then (36) is expressed as follows:

$$
\mathbf{X}=\mathbf{A} \mathbf{L}
$$

$$
\begin{aligned}
\mathbf{A} & =\left[a_{i j}\right]_{n \times n} \\
a_{i j} & =\frac{\omega_{i}}{1-\left(\omega_{i} / \omega_{r j}\right)^{2}} .
\end{aligned}
$$

The inductance vector is determined by

$$
\mathbf{L}=\mathbf{A}^{-1} \mathbf{X} \text {. }
$$

The required capacitances can be determined by applying inductances obtained by (43) into (37). Thus, the L-C network satisfying reactances at given frequencies is synthesized.

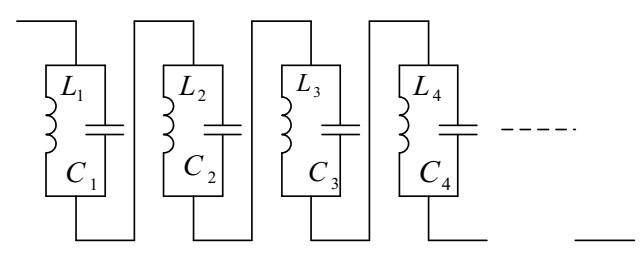

(a)

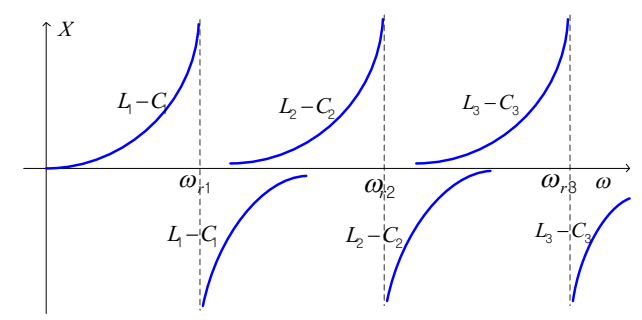

(b)

Fig. 6. Series-connected L-C parallel circuits

(a) circuit diagram, (b) its reactance variation

\section{Illustrative examples}

Two examples are given to show the effectiveness of the proposal. The first one shows an application of the series-parallel compensator to a power system with a nonlinear load. The second example shows an application of the L-C network to a system with a thyristor-controlled rectifier, where harmonic isolation is needed.

\subsection{Compensation when nonlinear impedance is given}

As a first example, a single-phase system is considered. The fundamental frequency is $60 \mathrm{~Hz}$, and 3rd, 5th, and 7th harmonics are included. The line voltage is given as follows:

$$
\begin{aligned}
v(t)=\sqrt{2} & \left(400 \cos \left(\omega t-15^{\circ}\right)+40 \cos \left(3 \omega t-45^{\circ}\right)\right. \\
+ & \left.+20 \cos \left(5 \omega t-15^{\circ}\right)+4 \cos \left(7 \omega t+65^{\circ}\right)\right) .
\end{aligned}
$$

The average power of the load is 42 [kW], and the load admittance is given in Table I. Let us express the line voltages as the following vector:

$$
\mathbf{V}_{\mathrm{s}}=\left[\begin{array}{lll}
400 \angle-15^{\circ} \quad 40 \angle-45^{\circ} \quad 20 \angle-15^{\circ} \quad 4 \angle 65^{\circ}
\end{array}\right]^{T} .
$$

Each entry is a phasor with rms magnitude, and its frequency is given in the corresponding entry of the following frequency vector:

$$
\boldsymbol{\Omega}=2 \pi\left[\begin{array}{llll}
60 & 180 & 300 & 420
\end{array}\right]^{T} .
$$

where 
Table I. Admittances of load

\begin{tabular}{|c|c|c|c|c|}
\hline Harm. order & 1 & 3 & 5 & 7 \\
\hline$G_{L}^{\omega_{i}}$ & 0.25 & 0.225 & 0.2 & 0.25 \\
\hline$B_{L}^{\omega_{i}}$ & 0.25 & -0.125 & 0.125 & 0.2 \\
\hline
\end{tabular}

For unity power factor, the power should be distributed according to (9) and the source current is given according to (10) as follows:

$$
\begin{aligned}
& \mathbf{P}_{\mathrm{s}}=\left[\begin{array}{llll}
41.4774 & 0.4148 & 0.1037 & 0.0041
\end{array}\right]^{T} \mathrm{~kW} \\
& \mathbf{I}_{\mathrm{s}}=\left[\begin{array}{llll}
103.69 \angle-15^{\circ} & 10.37 \angle-45^{\circ} & 5.18 \angle-15^{\circ} & 1.04 \angle 65^{\circ}
\end{array}\right]^{T} .
\end{aligned}
$$

From (13), the reactance of the series component is given by

$$
\mathbf{X}_{\mathrm{S}}=\left[\begin{array}{llll}
0.7414 & 1.5047 & 2.0993 & 0.7414
\end{array}\right]^{T} \quad \Omega .
$$

The load voltage is given by

$$
\begin{array}{r}
\mathbf{V}_{\mathrm{L}}=\left[\begin{array}{ll}
407.32 \angle-25.88^{\circ} & 42.94 \angle-66.31^{\circ} \\
22.77 \angle-43.56^{\circ} & 4.07 \angle 54.12^{\circ}
\end{array}\right]^{\mathrm{T}} .
\end{array}
$$

The load current is given by

$$
\begin{array}{r}
\mathbf{I}_{\mathrm{L}}=\left[\begin{array}{cc}
144.01 \angle 19.12^{\circ} & 11.05 \angle-95.36^{\circ} \\
5.37 \angle-11.55^{\circ} & 1.30 \angle 92.78^{\circ}
\end{array}\right]^{T} .
\end{array}
$$

The required compensation current is as follows:

$$
\begin{gathered}
\mathbf{I}_{\mathrm{C}}=\left[\begin{array}{ll}
82.26 \angle 64.12^{\circ} & 9.14 \angle-156.31^{\circ} \\
0.37 \angle 46.44^{\circ} & 0.62 \angle 144.12^{\circ}
\end{array}\right]^{T} .
\end{gathered}
$$

From (17), the desired susceptances of the parallel element are given as entries of the following susceptance vector:

$$
\mathbf{B}_{\mathrm{P}}=\left[\begin{array}{lll}
-0.2020 & 0.2128 & -0.0162-0.1520
\end{array}\right]^{\mathrm{T}} .
$$

Thus, reactances and susceptances for series and parallel elements are obtained. Next, we will synthesize L-C networks satisfying the required reactances and susceptances.

The procedure (30)-(35) can be applied to get appropriate components, after negating and taking inverses of reactance vector $\mathbf{X}_{\mathrm{s}}$ given in (49):

$$
\mathbf{B}_{\mathrm{S}}=\left[\begin{array}{lll}
-1.3489-0.6646-0.4763-1.3489
\end{array}\right]^{T} \mathrm{~S} .
$$

The resonance frequencies for 4 series L-C circuits are selected as follows:

$$
\boldsymbol{\Omega}_{\text {res_s }}=2 \pi\left[\begin{array}{llll}
57 & 170 & 290 & 410
\end{array}\right]^{T} .
$$

The matrix in (33) is constructed as follows:

$$
\mathbf{A}_{\mathrm{S}}=\left[\begin{array}{rrrr}
-3490 & 431 & 394 & 385 \\
-126 & -9339 & 1840 & 1401 \\
-71 & -892 & -26869 & 4057 \\
-50 & -517 & -2404 & -53446
\end{array}\right] \text {. }
$$

The required capacitances are given by

$$
\mathbf{C}_{\mathrm{S}}=\mathbf{A}_{\mathrm{S}}^{-1} \mathbf{B}_{\mathrm{S}}=\left[\begin{array}{llll}
400.1 & 72.8 & 17.8 & 23.4
\end{array}\right]^{T} \quad \mu \mathrm{F} .
$$

According to (29), the required inductances are given by

$$
\mathbf{L}_{\mathrm{S}}=\left[\begin{array}{llll}
19.49 & 12.04 & 16.93 & 6.45
\end{array}\right]^{T} \mathrm{mH} .
$$

The circuit of the series element designed with parallel L$\mathrm{C}$ circuits is shown in Fig. 7.

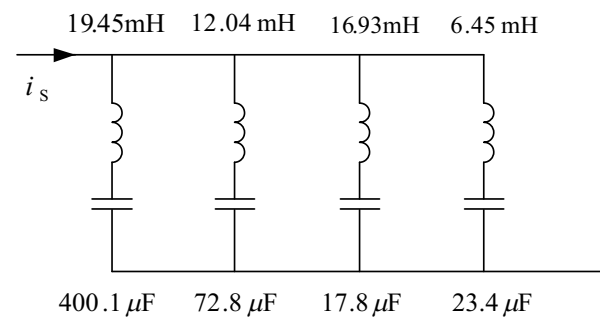

Fig. 7. Implementation of series element with series L-C circuits in parallel.

The parallel element can be implemented with 4 parellel L-C circuits satisfying the susceptances given by (53). The procedure (38)-(43) can be applied to get appropriate components, after negating and taking inverses of reactance vector $\mathbf{B}_{P}$ given in (53):

$$
\mathbf{X}_{\mathrm{P}}=\left[\begin{array}{llll}
4.9516 & -4.7000 & 61.8915 & 6.5809
\end{array}\right]^{\mathrm{T}} .
$$

Their resonance frequencies are selected as follows:

$$
\boldsymbol{\Omega}_{\text {res_P }}=2 \pi\left[\begin{array}{lllll}
70 & 160 & 310 & 450
\end{array}\right]^{T} .
$$

The required capacitances and inductances are given by

$$
\begin{aligned}
& \mathbf{C}_{\mathrm{P}}=\left[\begin{array}{llll}
2467.0 & 482.4 & 127.7 & 180.6
\end{array}\right]^{T} \quad \mu \mathrm{F} \\
& \mathbf{L}_{\mathrm{P}}=\left[\begin{array}{lllll}
2.095 & 2.051 & 2.065 & 0.693
\end{array}\right] \mathrm{mH} .
\end{aligned}
$$

The circuit of the parallel element designed with series L$\mathrm{C}$ circuits is shown in Fig. 8.

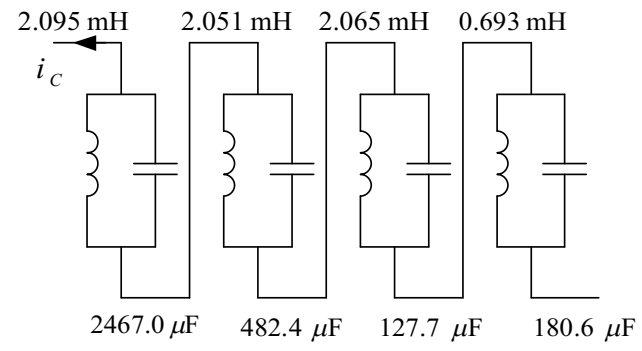

Fig. 8. Implementations of parallel element with parallel L-C circuits in series.

\subsection{Harmonic isolation}

As a second example, a power system with a thyristor controlled rectifier is considered. The line voltage is sinusoidal and its rms value is $220 \mathrm{~V}$. The load current of the thyristor rectifier is $20 \mathrm{~A}$ dc. The rectifier's firing angle is 30 degrees. The fundamental frequency is $60 \mathrm{~Hz}$. Only the 3rd, 5th and 7th harmonics are considered for a simplicity. Although a parallel element can compensate reactive power, a series-parallel compensator is considered. Its series element blocks harmonic voltages which can exist in an actual situation. Its parallel element traps harmonic currents. The line voltage is expressed as follows:

$$
\mathbf{V}_{\mathrm{s}}=\left[\begin{array}{lllll}
220 \angle 0^{\circ} & 0 \angle 0^{\circ} & 0 \angle 0^{\circ} & 0 \angle 0^{\circ} & 0 \angle 0^{\circ}
\end{array}\right]^{T}
$$


The load current is given as follows:

$$
\mathbf{I}_{\mathrm{L}}=\frac{440 \sqrt{2}}{\pi}\left[1 \angle 30^{\circ} \quad \frac{1}{3} \angle-90^{\circ} \quad \frac{1}{5} \angle-150^{\circ} \quad \frac{1}{7} \angle-210^{\circ}\right]^{T} .
$$

Each entry is rms phasor whose frequency is given in the corresponding entry of the frequency vector given by

$$
\boldsymbol{\Omega}=2 \pi\left[\begin{array}{llll}
60 & 180 & 300 & 420
\end{array}\right]^{T} .
$$

The required reactance of the series element is given by

$$
\mathbf{X}_{\mathrm{S}}=\left[\begin{array}{llll}
0 & \infty & \infty & \infty
\end{array}\right]^{T} \quad \Omega .
$$

The infinite reactance means that the series element blocks the corresponding harmonic voltage. The reactive components could not be calculated through procedure (38)-(43), because its computation could not be performed with infinite numbers. A parallel resonant circuit can be used for each infinite reactance. After three resonant circuits are designed, a single capacitor or inductor can be selected for satisfying reactance for fundamental frequency. The required capacitances and inductances are given by

$$
\begin{aligned}
& \mathbf{C}_{\mathrm{S}}=\left[\begin{array}{llll}
202.8 & 50.0 & 30.0 & 20.0
\end{array}\right]^{T} \quad \mu \mathrm{F} \\
& \mathbf{L}_{\mathrm{S}}=\left[\begin{array}{llll}
\infty & 15.64 & 9.38 & 7.18
\end{array}\right]^{T} \mathrm{mH} .
\end{aligned}
$$

The susceptances of the parallel element are given by

$$
\mathbf{B}_{\mathrm{P}}=\left[\begin{array}{llll}
0.0409 & \infty & \infty & \infty
\end{array}\right]^{\mathrm{T}} .
$$

The reactive components could not be given through procedure (30)-(35), because its computation could not be performed with infinite numbers. A series tuned resonant circuit can be used for each infinite susceptance. After three resonant circuits are designed, a single capacitor or inductor can be selected for satisfying susceptance for the fundamental frequency. $10 \mu \mathrm{F}, 5 \mu \mathrm{F}$ and $3 \mu \mathrm{F}$ are selected for the series L-C circuits resonating at the 3rd, 5th and 7 th harmonics, respectively. The required inductances for resonances are given by

$$
\mathbf{L}_{\mathrm{P}}=\left[\begin{array}{llll}
0.0 & 78.2 & 56.3 & 47.9
\end{array}\right]^{T} \quad \mathrm{mH} \text {. }
$$

Capacitance of $89 \mu \mathrm{F}$ is selected for achieving $0.0409 \mathrm{~S}$ at the fundamental frequency including 3 resonant circuits.

$$
\mathbf{C}_{\mathrm{P}}=\left[\begin{array}{llll}
89.0 & 10.0 & 5.0 & 3.0
\end{array}\right]^{T} \quad \mu \mathrm{F} .
$$

The designed circuit is shown in Fig. 9.

\section{Conclusion}

In this paper, passive series-parallel compensation is studied. The series-parallel compensation is useful in achieving high power factor by compensating a nonlinear load whose impedances are given at specified frequencies, and in isolating a power source from a load with respect to harmonics. An easy method to synthesize L-C circuits to have specified reactances or susceptances at predefined frequencies is proposed. It is expected to contribute in power factor correction and harmonic isolation.

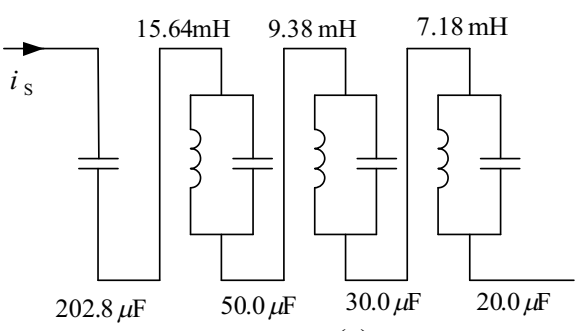

(a)

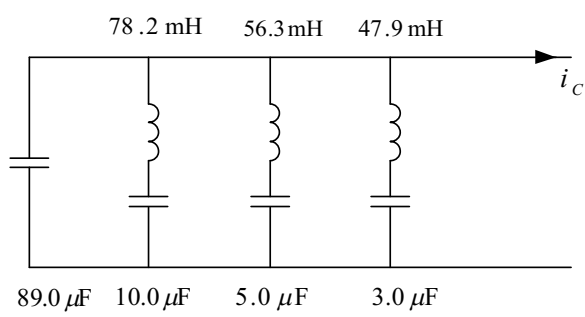

(b)

Fig. 9. Series-parallel compensation network:

(a) series element, (b) parallel element

\section{References}

[1] W.V. Lyon, "Reactive power and unbalanced circuits," Electrical World, vol. 75, no. 25, pp. 1417-1420, June 1920.

[2] T.H.E. Miller, Reactive Power Control in Electric Systems, John Wiley \& Sons: New York, 1982.

[3] W. Shepherd and P. Zakikhani, "Suggested definition of reactive power for nonsinusoidal systems," IEE Proc., vol. 119, no. 9, pp. 1361-1362, 1972.

[4] D. Sharon, "Reactive power definition and power-factor improvement in nonlinear systems," IEE Proc., vol. 120, no. 6, pp. 704-706, 1973.

[5] N.L. Kusters and W.J.M. Moore, "On the definition of reactive power under non-sinusoidal conditions," IEEE Trans. Power Apparatus and Systems, vol. 99, no. 5, pp. 1845-1854, 1980.

[6] M.M.A. Aziz, E.E.A. El-Zahab, A.M. Ibrahim and A.F. Zobaa, "LC compensator for power factor correction of nonlinear loads," IEEE Trans. Power Delivery, vol. 19, no. 1, pp. 331-335, 2004

[7] D. Sharon, "Power factor definitions and power transfer quality in nonsinusoidal situations," IEEE Trans. Instrumentation and Measurement, vol. 45, no. 3, pp. 728733, 1996.

[8] C.S.A. Mboving, Z. Hanzelka and A. Mondzik, "Different LC power filter topologies - effectiveness of reducing voltage distortion," $R E \& P Q J$, no. 14, pp. 332-327, May 2016.

[9] L.S. Czarnecki, "Scattered and reactive current, voltage, and power in circuit with nonsinusoidal waveforms and their compensation," IEEE Trans. Instr. and Meas., vol. 40, no. 3, pp. 563-567, 1991.

[10] R. Sasdelli and G.C. Montanari, "Compensable power for electrical systems in nonsinusoidal conditions," IEEE Trans. Instr. and Meas., vol. 43, no. 4, pp. 592-598, 1994.

[11] N. LaWhite and M.D. Ilic, "Vector space decomposition of reactive power for periodic nonsinusoidal signals," IEEE Trans. Circuits and Systems, vol. 44, no. 4, pp. 338-346, 1997.

[12] S.-J. Jeon, "Considerations on a reactive power concept in a multi-line system," IEEE Trans. Power Delivery, vol. 21, no. 2, pp. 551-559, Apr. 2006. 